\title{
USO DE IMAGENS LANDSAT-7 ETM+ PARA O MAPEAMENTO DE PLANTAÇÕES DE Eucalyptus NA REGIÃO NORTE DE MINAS GERAIS
}

\author{
Vicente Paulo Soares*, Wellington Donizete Guimarães**, Carlos Antonio Álvares Soares Ribeiro***, \\ Antonio Simões Silva**** \\ *Eng. Florestal, Dr., Depto. de Engenharia Florestal, UFV - vicente@ufv.br \\ ** Professor M.Sc., Escola Técnica Federal, UFES - wdguimaraes@cefetes.br \\ ***Eng. Agrícola, Dr., Departamento de Engenharia Florestal, UFV - cribeirioi@ufv.br \\ **** Eng. Cartográfico, Dr., Depto. de Engenharia Civil, UFV - asimoes@ufv.br \\ Recebido para publicação: 12/10/2004 - Aceito para publicação: 16/03/2005
}

\begin{abstract}
Resumo
Uso de imagens LANDSAT-7 etm + para o mapeamento de plantações de Eucalyptus na região norte de Minas Gerais. O presente trabalho foi realizado em uma área de plantios de Eucalyptus de propriedade da Acesita Energética Ltda. Os plantios estão localizados nos municípios de Turmalina, Minas Novas, Veredinha e Capelinha, em Minas Gerais. O estudo teve como objetivo avaliar a viabilidade técnica da adoção de imagens Landsat-7 ETM+ para o mapeamento dos talhões de eucalipto. Foram empregadas duas metodologias de digitalização, em mesa e em tela. Duzentos e cinqüenta e quatro talhões foram digitalizados e a área de cada talhão foi calculada e comparada com os valores tomados como referência, fornecidos pela empresa. Como principais resultados citam-se: na digitalização em mesa, $43,30 \%$ dos talhões apresentaram erros na faixa de 0 a $5 \%$ e $64,95 \%$ na faixa de 0 a $10 \%$, enquanto que na digitalização em tela os valores encontrados foram, respectivamente, $48,43 \%$ e $70,48 \%$; não se aconselha o mapeamento de divisas com essa metodologia, uma vez que elas requerem maior exatidão; levantamentos expeditos ou de reconhecimento podem ser realizados com qualquer uma das metodologias.
\end{abstract}

Palavras-chave: Imagem Landsat-TM; eucalipto; sensoriamento remoto.

\begin{abstract}
Use of orbital images for mapping Eucalyptus forest plantations in the north region of Minas Gerais state, Brazil. The present work was carried out in an eucalypt stand area, belonging to the Acesita Energética Ltda. The stands are located in the municipalities of Turmalina, Minas Nova, Veredinha and Capelinha, Minas Gerais State, Brazil. The objective of this study was to evaluate the technical suitability of adoption of Landsat TM-7+ data for mapping Eucalyptus stands. Two digitalization methodologies were used, on digitizing tablet and screen. A total of 254 stands were digitalized and the area of each stand was calculated and compared with values taken as reference, provided by the Acesita Ltda. The main results were: on the tablet digitalization, $43.30 \%$ of the stands presented errors in the range of 0 to $5 \%$ and $64.95 \%$ in the range of 0 to $10 \%$, while on the screen digitalization the values found were, respectively, $48.43 \%$ and $70.48 \%$. It is not recommended to map limits with this methodology since they require a greater accuracy; expedite or recognizing surveys can be carried out with either methodology.

Keywords: Landsat TM image; Eucalyptus; remote sensing.
\end{abstract}

\section{INTRODUÇÃO}

A crescente demanda do mercado por produtos que conciliem qualidade e preço impõe às empresas uma procura constante por alternativas tecnológicas que possam agregar valor aos seus produtos e otimizar todos os setores do seu processo produtivo. Em se tratando de empresas do setor florestal, uma atividade que exige o monitoramento contínuo se refere ao inventário de suas áreas plantadas, haja visto o 
seu caráter dinâmico, caracterizado pela variabilidade de estágios de desenvolvimento e de espécies de cada talhão, áreas afetadas por incêndios, pragas, controle de corte e plantio, entre outros fatores.

Estas características, inerentes aos plantios de Eucalyptus, inviabilizam o gerenciamento de suas atividades apenas com um banco de dados descritivo, sendo de suma importância o componente espacial da informação, que, juntos, compõem um Sistema de Informações Geográficas. Este permitirá processar informações espaciais, devendo ser capaz de criar abstrações digitais do real, manejar e armazenar eficientemente dados, de forma a identificar o melhor relacionamento entre as variáveis espaciais, possibilitando a criação de relatórios e mapas que contribuem para a compreensão holística desses relacionamentos (Ribeiro et al., 2000). Os resultados decorrentes dos processos de tomada de decisão irão depender, em grande parte, da confiabilidade e adequabilidade desta base de dados, sendo que, segundo Ribeiro et al. (2000), de uma maneira geral, $75 \%$ do orçamento e do tempo de um projeto nessa área são consumidos na fase de sua elaboração.

Uma metodologia usualmente empregada no mapeamento geodésico de áreas utiliza o Sistema de Posicionamento Global (GPS). Embora esta técnica atenda aos requisitos geométricos de exatidão, ela pode ser onerosa, e principalmente, não conter a riqueza de detalhes e de informações presentes em uma imagem orbital de alta resolução, como por exemplo, uma imagem do satélite Ikonos - 1 metro no modo pancromático. Estas, apresentam como inconvenientes, o custo elevado e a extensão relativamente pequena das áreas imageadas, contrastando com as extensas áreas cobertas pelos povoamentos. Isto implica numa relação benefício/custo inadequada para o setor.

Como alternativa, pode-se avaliar a utilização de imagens de menor resolução espacial (15 m e $20 \mathrm{~m}$ ), que podem cobrir grandes extensões do terreno. Mesmo com esta resolução, as imagens ainda conterão informações importantes não apenas para o levantamento geodésico das áreas, mas também para suporte ao planejamento, auxiliando no processo de tomada de decisão. Desta forma, pode-se disponibilizar informações sobre o erro cometido nas determinações de áreas, para que o tomador de decisão verifique se estes estão dentro de limites aceitáveis para o seu propósito.

Inúmeros trabalhos vêm sendo feitos com imagens Landsat nas últimas três décadas para o mapeamento e monitoramento da cobertura terrestre, sendo alguns citados a seguir:

Soares e Hoffer (1996) utilizaram imagens TM/Landsat para monitorar mudanças em plantações de Eucalyptus, através da diferença entre bandas, de imagens obtidas em épocas diferentes. Skidmore (1989) desenvolveu um sistema especialista para classificar tipos de florestas de Eucalyptus. Além dos dados do mapeador temático do Landsat, o sistema incorporou informações derivadas do modelo digital de elevação - declividade e orientação - e o conhecimento de campo do responsável pela área para estabelecer as regras norteadoras do processo de classificação.

Outra aplicação importante das imagens orbitais é a atualização de mapas. Diversos órgãos públicos, como IEF, IBAMA e INPE, utilizam-se desse expediente para monitorar queimadas e desmatamentos.

Sartori Neto (2000) utilizou imagens TM/Landsat-5 para gerar o mapa de vegetação do Parque Nacional Grande Sertão Veredas (noroeste do estado de Minas Gerais), que compôs a base de dados necessária para elaboração do plano de manejo desse parque.

Medeiros et al. (1996) utilizaram imagens TM/Landsat-5 para estimar áreas de plantio de canade-açúcar, milho e soja, nos municípios de Aramina, Buritizal, Ituverava e Ipuã, todos pertencentes ao estado de São Paulo.

Face às considerações anteriores, o objetivo principal deste trabalho foi mapear e quantificar áreas ocupadas por plantações de Eucalyptus, através de imagens dos satélites Landsat-7 ETM+.

\section{MATERIAL E MÉTODOS}

A área de estudo localiza-se nos municípios de Turmalina, Minas Novas, Veredinha e Capelinha, estado de Minas Gerais, sendo parte de uma fazenda de propriedade da ACESITA ENERGÉTICA LTDA, com uma área de aproximadamente 6.829 ha, que se encontra entre as latitudes $17^{\circ} 17^{\prime} \mathrm{S}$ e $17^{\circ}$ $27^{\prime} \mathrm{S}$ e longitudes $42^{\circ} 44^{\prime} \mathrm{W}$ e $42^{\circ} 33^{\prime} \mathrm{W}$. Segundo Golfari (1975), a região compreende chapadões baixos e colinas com relevo ondulado suave a ondulado forte, com altitude variando entre $600 \mathrm{~m}$ e 1000 $\mathrm{m}$. As plantações de Eucalyptus localizadas nesta área são manejadas para a produção de carvão vegetal e de móveis, com rotação média de sete anos. 
Como fonte de consulta, foi utilizado uma carta plani-altimétrica do IBGE, denominada Minas Novas, escala 1:100.000 e uma imagem digital do satélite LANDSAT-7 ETM+, fusão das bandas 5, 4, 3 e pan, de 18 de maio de 2000, órbita-ponto 217_072, com resolução espacial de $15 \mathrm{~m}$. Também foi utilizada uma imagem impressa em papel fotográfico, escala 1:50.000, correspondente à cena da imagem LANDSAT-7 ETM+.

Para o trabalho de campo, realizado no mês de maio de 2001, utilizou-se um receptor GPS ProXLS com precisão de 0,5 metros para coletar as coordenadas geográficas dos potenciais pontos de controle terrestre, para a posterior correção geométrica das imagens orbitais e georreferenciamento da carta-imagem.

$\mathrm{Na}$ execução dos trabalhos de laboratório, utilizou-se o software SPRING 3.4 para o processamento das imagens orbitais e a digitalização em tela. A elaboração dos mapas e a manipulação dos atributos de cada talhão foram realizados no software ArcView 3.2a, apoiado em uma mesa digitalizadora formato A0 (SumaGraphics ${ }^{\circledR}$, modelo Summagrid ${ }^{\text {TM }}$ III).

\section{Dados de referência}

Como verdade de campo, foi adotado um mapeamento realizado pela ACESITA ENERGÉTICA LTDA, contendo 254 talhões, realizado com o GPS ProXLS, com tempo de permanência por ponto de 15 segundos, cujos dados sofreram um pós-processamento aplicando-se a correção diferencial. O arquivo fornecido pela empresa foi editado no AutoCad, para remoção de eixos de estradas e linhas duplicadas, deixando apenas os limites dos talhões. Posteriormente, este arquivo foi exportado para o ArcView, onde se calculou a área correspondente a cada talhão, sendo este arquivo usado posteriormente para avaliação dos outros métodos.

\section{Determinação das áreas}

\section{Digitalização em mesa}

Na mesa digitalizadora realizou-se a vetorização dos 254 talhões de Eucalyptus utilizando a imagem Landsat-7 ETM+, sendo o georreferenciamento realizado a priori, em cada sessão de digitalização. Paralelamente, atribuiu-se um identificador para cada um dos talhões. Em seguida, fez-se a edição e a criação da topologia de polígonos. O arquivo resultante foi exportado para o ArcView, onde se calculou a área de cada talhão. A Figura 1 mostra o mapeamento dos 254 talhões resultante da digitalização em mesa

\section{Digitalização em tela}

A primeira etapa foi georreferenciar a imagem. Nesta etapa foram utilizados 21 pontos de controle, distribuídos uniformemente sobre a área. Utilizou-se uma equação polinomial de primeira ordem. O método de interpolação de intensidade adotado foi o do Vizinho Mais Próximo. Ao término da digitalização, realizou-se a edição, criação de topologia e identificação dos polígonos, conforme a numeração adotada na digitalização em mesa. O arquivo gerado foi exportado para o ArcView, onde as áreas dos talhões foram calculadas. A Figura 2 mostra o mapeamento dos talhões resultante da digitalização em tela da imagem Landsat-7 ETM+. 


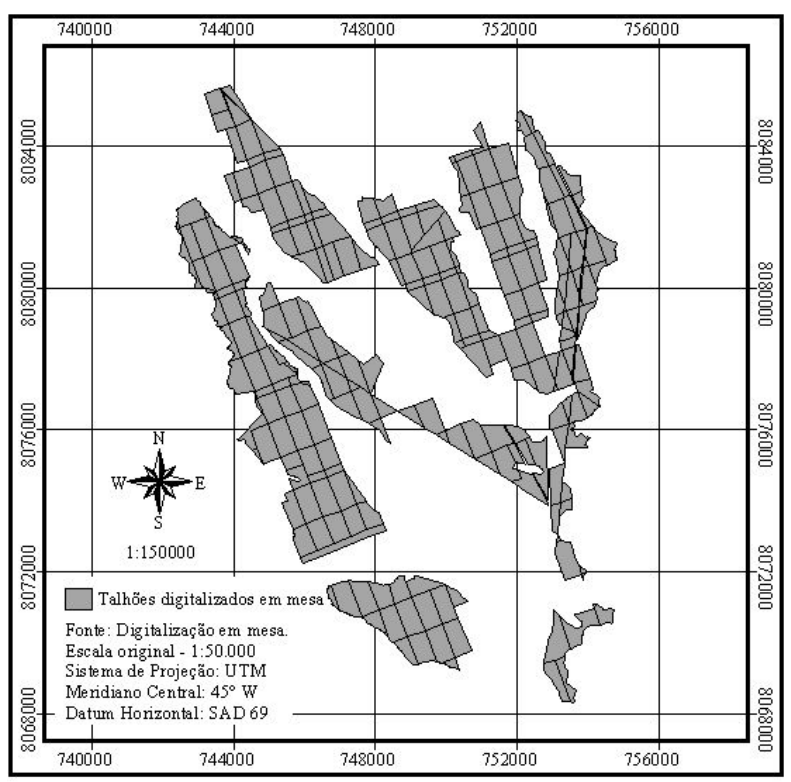

Figura 1. Mapa dos 254 talhões, resultante da digitalização em mesa da imagem Landsat-7 ETM+. Figure 1. Map of 254 stands, generated from digitizing tablet using Landsat-7 ETM+ data.

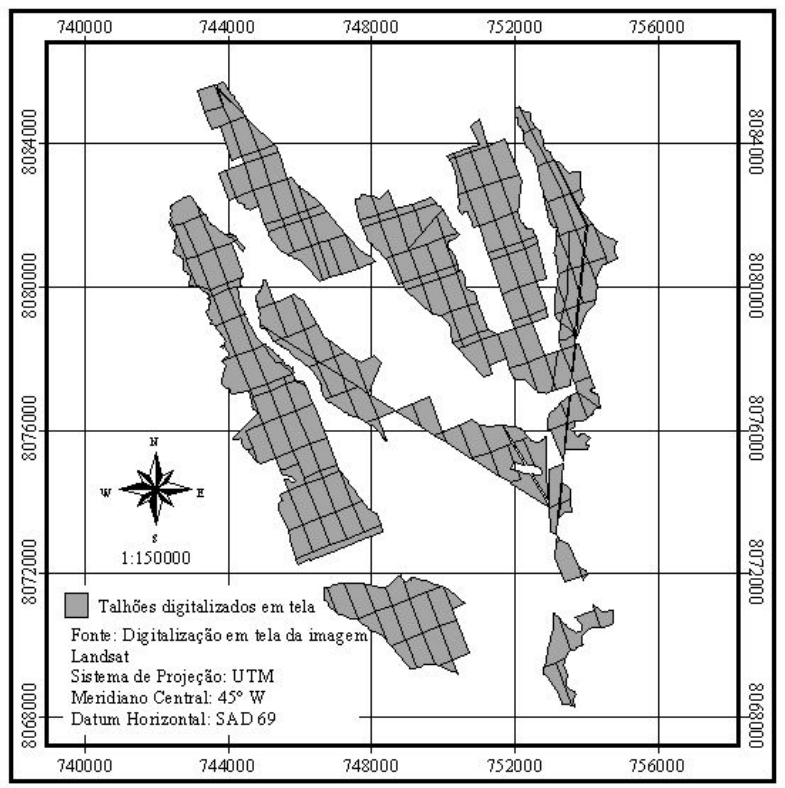

Figura 2. Mapa dos 254 talhões, resultante da digitalização em tela da imagem Landsat-7 ETM+. Figure 2. Map of 254 stands, generated from on screen digitizing using Landsat-7 ETM+ data. 


\section{Análise dos dados}

Classes de talhões

No decorrer das atividades de vetorização, tanto em mesa quanto em tela, verificou-se um nível diferenciado de dificuldade na identificação dos limites dos talhões, devido a variação do contraste entre estrada e cobertura vegetal, decorrentes do grau de uso e conservação das estradas, tombamento de árvores devido a vendavais e a presença de grande quantidade de veredas. Conseqüentemente, os talhões foram divididos em classes, conforme indica a Tabela 1.

Tabela 1. Classes de talhões classificados quanto à disposição espacial.

Table 1. Stand classes classified based on their spatial position.

\begin{tabular}{c|c|c}
\hline Classes & Descrição & Número \\
\hline 1 & Talhões internos & 103 \\
\hline 2 & Talhões de borda & 151 \\
\hline Total & & 254 \\
\hline
\end{tabular}

Além do contraste, as classes também contemplaram geometrias diferenciadas. A classe 1 apresenta talhões retangulares ou triangulares, enquanto a 2 possui talhões de formas irregulares.

Cálculo dos erros

Os arquivos provenientes das digitalizações, juntamente com o mapeamento tomado como referência, foram importados para o ArcView, onde se calculou a área dos talhões. Cada talhão recebeu um identificador, através do qual pôde-se transferir os valores de área do mapa fornecido pela ACESITA para cada uma das tabelas de atributos dos temas digitalizados. Em seguida, calculou-se o erro percentual cometido, segundo a equação 1 .

$$
\operatorname{Erro}(\%)=\frac{(\text { Área Digitalizada }- \text { Área de Referência })}{\text { Área de Referência }} * 100 \quad \text { Equação } 1
$$

Agrupamento dos talhões em classes de erro e área

Para avaliar o comportamento do erro, os talhões foram agrupados em 36 classes de erros, com amplitude de 5\%, para as digitalizações em mesa e tela das imagens Landsat-7 ETM+. Foram ainda gerados os gráficos relacionando o erro e a área de cada um dos talhões, as áreas de referência e as digitalizadas.

\section{RESULTADOS E DISCUSSÕES}

\section{Digitalização da carta-imagem Landsat-7 em mesa digitalizadora}

A vetorização dos talhões da carta-imagem foi realizada em 5 seções de digitalização, tendo sido utilizados para o georreferenciamento, em média, 23 pontos de controle terrestres, com um RMS (Erro Médio Quadrático) de 8,8 m.

A Figura 3 fornece o comportamento geral dos erros em relação ao tamanho das áreas dos talhões, através de um diagrama de dispersão, útil para se verificar a associação entre variáveis quantitativas. Nela, estão representados os erros de todos os 254 talhões digitalizados. Considerando-se seus valores absolutos, pode-se verificar que os maiores erros estão associados aos menores talhões, seguindo uma tendência de queda e de estabilização à medida que a área aumenta. Observa-se também uma tendência de superestimar áreas, onde 202 talhões (79,5\%) apresentaram erros positivos, conforme constatado pela Tabela 2. Isto ocorre porque os talhões de referência desconsideram as áreas das estradas, enquanto a digitalização, dada a resolução espacial do sensor e a largura das estradas (em geral, $10 \mathrm{~m}$ para os talhões internos e $20 \mathrm{~m}$ para os de borda), não permite este nível de detalhamento. 


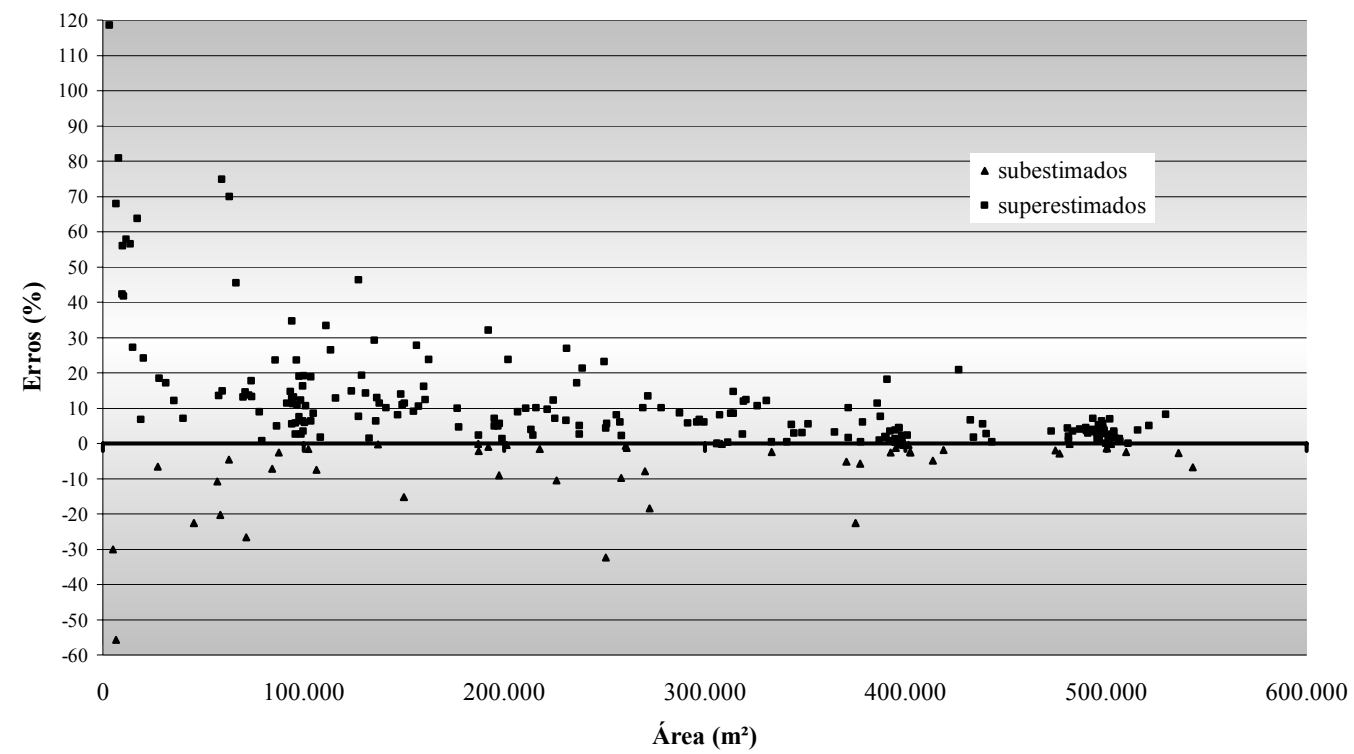

Figura 3. Diagrama de dispersão dos erros cometidos na digitalização da imagem Landsat usando mesa digitalizadora, considerando todos os 254 talhões.

Figure 3. Scattering diagram of digitizing errors for all 254 stands using Landsat imagery and digitizing tablet.

No caso dos talhões de borda, embora eles tenham, em geral, estradas mais largas compondo parte de sua delimitação, elas apresentam uma maior quantidade de vegetação rasteira, o que influencia na resposta espectral captada pelo sensor, diminuindo o contraste entre estrada e vegetação e, conseqüentemente, dificultando a digitalização. Outro comportamento importante é a alta dispersão da variável erro em relação a pequenos intervalos da variável área, indicando que o tamanho dos talhões não é o único fator que influencia os erros. Dentre outros fatores que contribuíram para o aumento do erro, citam-se a presença de sombras e estradas cobertas devido ao tombamento de árvores. Quando se consideram os talhões separados por classes, conforme indicam as Figuras 4 e 5, observa-se um comportamento um pouco distinto, com os talhões internos apresentando um padrão de erros mais homogêneo em relação ao padrão apresentado pelos talhões de borda. Conforme a Tabela 2, dos 103 talhões classificados como internos, a área de 77 deles $(74,8 \%)$ foi superestimada; $95(92,2 \%)$ apresentam área com $\pm 20 \%$ de erro. Dos 151 talhões classificados como de borda, a área de 125 deles $(82,8 \%)$ foi superestimada e $123(81,5 \%)$ apresentam área com $\pm 20 \%$ de erro. 


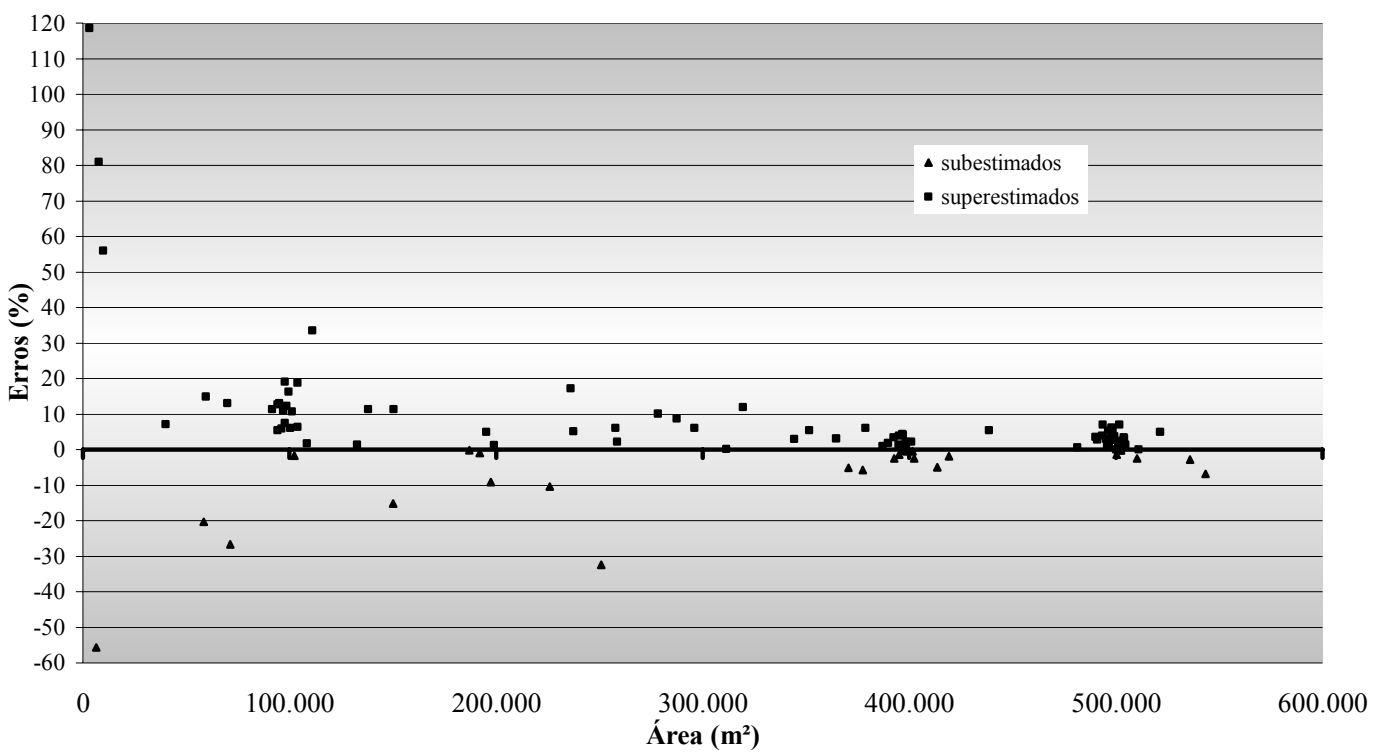

Figura 4. Diagrama de dispersão dos erros cometidos na digitalização dos talhões internos, usando imagem Landsat e mesa digitalizadora.

Figure 4. Scattering diagram of digitizing errors for all internal stands, using Landsat imagery and digitizing tablet.

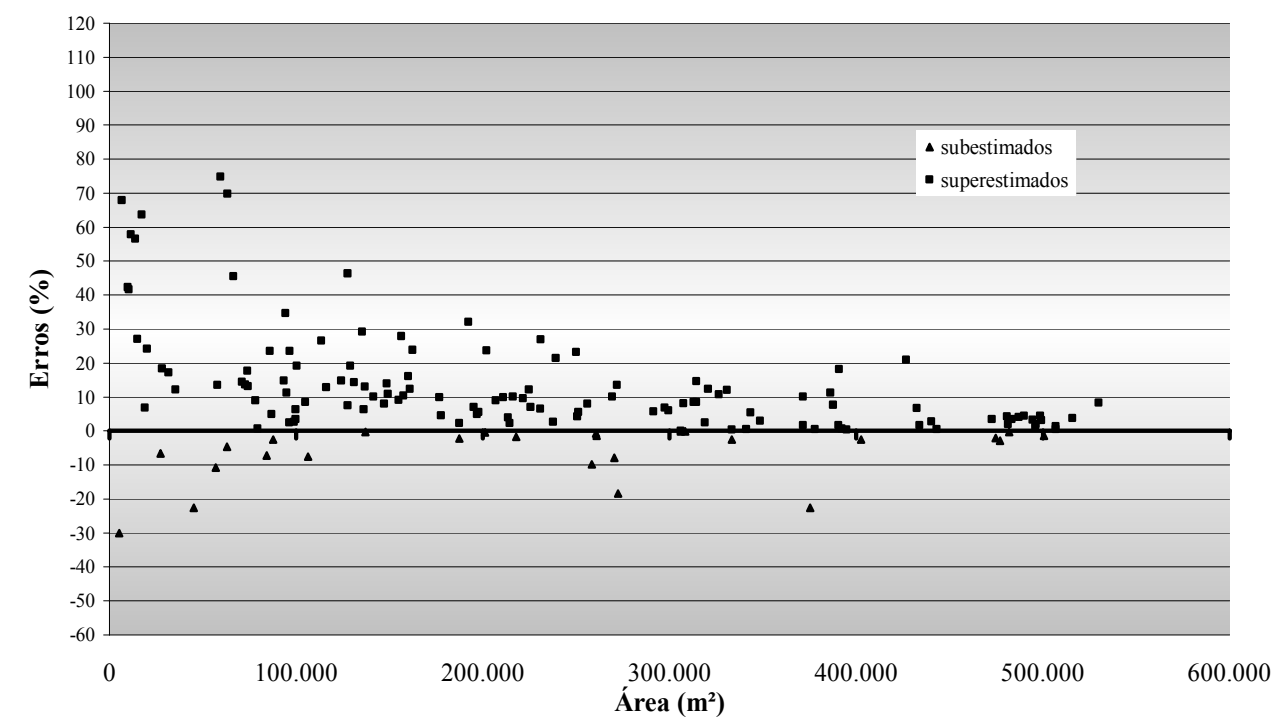

Figura 5. Diagrama de dispersão dos erros cometidos na digitalização dos talhões de borda, usando imagem Landsat e mesa digitalizadora.

Figure 5. Scattering diagram of digitizing errors for all bordering stands, using Landsat imagery and digitizing tablet.

A Tabela 2 mostra também uma grande concentração das freqüências no intervalo que vai de $10 \%$ a $20 \%$, tanto para os talhões como um todo ou subdivididos nas classes 1 e 2 , conforme sintetizado na Tabela 3, onde os valores foram tomados em módulo. Esta concentração pode ser justificada lembrando-se que as estradas foram tomadas como referência para a digitalização, sendo que há um 
padrão de largura por todo o projeto, independentemente do tamanho do talhão. A análise da Tabela 3 mostra que $43,30 \%$ dos talhões (110) estão na faixa de $0 \%$ a $5 \%$ de erros e $64,95 \%$ (165) estão na faixa de $0 \%$ a $10 \%$. Analisando por classe de talhão, os internos apresentam uma freqüência relativa de $53,40 \%$ (55) para o intervalo de $0 \%$ a $5 \%$ de erros e $74,76 \%$ (77) para o intervalo de $0 \%$ a $10 \%$. Já para os talhões de borda, as freqüências relativas foram de $36,42 \%$ (55) e $58,27 \%$ (88), respectivamente.

Tabela 2. Distribuição de freqüência dos erros em classes com amplitude de 5\%.

Table 2. Frequency distribution of errors in range class of $5 \%$.

\begin{tabular}{|c|c|c|c|c|c|c|c|c|}
\hline \multirow{2}{*}{\multicolumn{3}{|c|}{$\begin{array}{c}\text { Classes de Erro } \\
(\%)\end{array}$}} & \multicolumn{3}{|c|}{ Freqüência Absoluta } & \multicolumn{3}{|c|}{ Freqüência Relativa (\%) } \\
\hline & & & \multirow{2}{*}{$\begin{array}{c}\text { TI } \\
1\end{array}$} & \multirow{2}{*}{$\begin{array}{c}\text { TB } \\
0\end{array}$} & \multirow{2}{*}{$\begin{array}{c}\text { TOTAL } \\
1\end{array}$} & \multirow{2}{*}{$\begin{array}{c}\text { TI } \\
0,97\end{array}$} & \multirow{2}{*}{$\begin{array}{c}\text { TB } \\
0,00\end{array}$} & \multirow{2}{*}{$\begin{array}{c}\text { TOTAL } \\
0,39\end{array}$} \\
\hline-60 & $\mathrm{a}$ & -55 & & & & & & \\
\hline-55 & $\mathrm{a}$ & -50 & 0 & 0 & 0 & 0,00 & 0,00 & 0,00 \\
\hline-50 & $\mathrm{a}$ & -45 & 0 & 0 & 0 & 0,00 & 0,00 & 0,00 \\
\hline-45 & $\mathrm{a}$ & -40 & 0 & 0 & 0 & 0,00 & 0,00 & 0,00 \\
\hline-40 & $\mathrm{a}$ & -35 & 0 & 0 & 0 & 0,00 & 0,00 & 0,00 \\
\hline-35 & $\mathrm{a}$ & -30 & 1 & 1 & 2 & 0,97 & 0,66 & 0,79 \\
\hline-30 & $\mathrm{a}$ & -25 & 1 & 0 & 1 & 0,97 & 0,00 & 0,39 \\
\hline-25 & $\mathrm{a}$ & -20 & 1 & 2 & 3 & 0,97 & 1,32 & 1,18 \\
\hline-20 & $\mathrm{a}$ & -15 & 1 & 1 & 2 & 0,97 & 0,66 & 0,79 \\
\hline-15 & $\mathrm{a}$ & -10 & 1 & 1 & 2 & 0,97 & 0,66 & 0,79 \\
\hline-10 & $\mathrm{a}$ & -5 & 4 & 5 & 9 & 3,88 & 3,31 & 3,54 \\
\hline-5 & $\mathrm{a}$ & 0 & 16 & 16 & 32 & 15,53 & 10,60 & 12,60 \\
\hline 0 & $\mathrm{a}$ & 5 & 39 & 39 & 78 & 37,86 & 25,83 & 30,71 \\
\hline 5 & $\mathrm{a}$ & 10 & 18 & 28 & 46 & 17,48 & 18,54 & 18,11 \\
\hline 10 & $\mathrm{a}$ & 15 & 12 & 26 & 38 & 11,65 & 17,22 & 14,96 \\
\hline 15 & $\mathrm{a}$ & 20 & 4 & 7 & 11 & 3,88 & 4,64 & 4,33 \\
\hline 20 & $\mathrm{a}$ & 25 & 0 & 8 & 8 & 0,00 & 5,30 & 3,15 \\
\hline 25 & $\mathrm{a}$ & 30 & 0 & 5 & 5 & 0,00 & 3,31 & 1,97 \\
\hline 30 & $\mathrm{a}$ & 35 & 1 & 2 & 3 & 0,97 & 1,32 & 1,18 \\
\hline 35 & $\mathrm{a}$ & 40 & 0 & 0 & 0 & 0,00 & 0,00 & 0,00 \\
\hline 40 & $\mathrm{a}$ & 45 & 0 & 2 & 2 & 0,00 & 1,32 & 0,79 \\
\hline 45 & $\mathrm{a}$ & 50 & 0 & 2 & 2 & 0,00 & 1,32 & 0,79 \\
\hline 50 & $\mathrm{a}$ & 55 & 0 & 0 & 0 & 0,00 & 0,00 & 0,00 \\
\hline 55 & $\mathrm{a}$ & 60 & 1 & 2 & 3 & 0,97 & 1,32 & 1,18 \\
\hline 60 & $\mathrm{a}$ & 65 & 0 & 1 & 1 & 0,00 & 0,66 & 0,39 \\
\hline 65 & $\mathrm{a}$ & 70 & 0 & 2 & 2 & 0,00 & 1,32 & 0,79 \\
\hline 70 & $\mathrm{a}$ & 75 & 0 & 1 & 1 & 0,00 & 0,66 & 0,39 \\
\hline 75 & $\mathrm{a}$ & 80 & 0 & 0 & 0 & 0,00 & 0,00 & 0,00 \\
\hline 80 & $\mathrm{a}$ & 85 & 1 & 0 & 1 & 0,97 & 0,00 & 0,39 \\
\hline 85 & $\mathrm{a}$ & 90 & 0 & 0 & 0 & 0,00 & 0,00 & 0,00 \\
\hline 90 & $\mathrm{a}$ & 95 & 0 & 0 & 0 & 0,00 & 0,00 & 0,00 \\
\hline 95 & $\mathrm{a}$ & 100 & 0 & 0 & 0 & 0,00 & 0,00 & 0,00 \\
\hline 100 & $\mathrm{a}$ & 105 & 0 & 0 & 0 & 0,00 & 0,00 & 0,00 \\
\hline 105 & $\mathrm{a}$ & 110 & 0 & 0 & 0 & 0,00 & 0,00 & 0,00 \\
\hline 110 & $\mathrm{a}$ & 115 & 0 & 0 & 0 & 0,00 & 0,00 & 0,00 \\
\hline \multirow[t]{2}{*}{115} & $\mathrm{a}$ & 120 & 1 & 0 & 1 & 0,97 & 0,00 & 0,39 \\
\hline & & Total & 103 & 151 & 254 & 100,00 & 100,00 & 100,00 \\
\hline
\end{tabular}

$\mathrm{TI}=$ Talhão Interno

$\mathrm{TB}=$ Talhão de Borda 
Tabela 3. Síntese da Tabela 2, com os valores dos erros tomados em módulo.

Table 3. Synthesis of Table 2, with error values in module.

\begin{tabular}{|c|c|c|c|c|c|c|c|c|}
\hline \multirow{2}{*}{\multicolumn{3}{|c|}{$\begin{array}{c}\text { Classes de Erro } \\
(\%)\end{array}$}} & \multicolumn{3}{|c|}{ FreqüênciaAbsoluta } & \multicolumn{3}{|c|}{ Freqüência relativa (\%) } \\
\hline & & & \multirow{2}{*}{$\begin{array}{l}\mathrm{TI} \\
55\end{array}$} & \multirow{2}{*}{$\begin{array}{c}\mathrm{TB} \\
55\end{array}$} & \multirow{2}{*}{$\frac{\text { TOTAL }}{110}$} & \multirow{2}{*}{$\frac{\text { TI }}{53,40}$} & \multirow{2}{*}{$\frac{\mathrm{TB}}{36,42}$} & \multirow{2}{*}{$\frac{\text { TOTAL }}{43,30}$} \\
\hline 0 & $\mathrm{a}$ & 5 & & & & & & \\
\hline 5 & $\mathrm{a}$ & 10 & 22 & 33 & 55 & 21,36 & 21,85 & 21,65 \\
\hline 10 & $\mathrm{a}$ & 15 & 13 & 27 & 40 & 12,62 & 17,88 & 15,75 \\
\hline \multirow[t]{2}{*}{15} & a & 20 & 5 & 8 & 13 & 4,85 & 5,30 & 5,12 \\
\hline & & Total & 95 & 123 & 218 & 92,23 & 81,46 & 85,82 \\
\hline
\end{tabular}

$\mathrm{TB}=$ talhão de Borda

\section{Digitalização em tela da imagem Landsat-7}

No georreferenciamento da imagem obteve-se um RMS de 8,0 m. Comparando-se os resultados encontrados nas digitalizações da imagem Landsat, em tela e em mesa, observa-se um comportamento similar em ambos os casos. Assim, as considerações feitas para a digitalização em mesa podem ser extendidas para a digitalização em tela da imagem Landsat. Manteve-se uma tendência de superestimação das áreas, conforme ilustrado na Figura 6, que fornece o comportamento geral dos erros em relação ao tamanho das áreas dos talhões, através de um diagrama de dispersão. Considerando-se os valores absolutos, pode-se verificar que os maiores erros estão associados aos menores talhões, seguindo uma tendência de queda e de estabilização à medida que os valores da variável área aumentam. Conforme a Tabela 4, dos 254 talhões digitalizados, 196 (77,16\%) foram superestimados. Quando se considera as classes (internos e de borda), ocorrem pequenas variações em relação à digitalização da carta-imagem. Dos 103 talhões classificados como internos, a área de 81 deles $(78,6 \%)$ foi superestimada, conforme ilustra a Figura 7, enquanto que a área dos 151 talhões classificados como de borda, 115 deles $(76,2 \%)$ foi superestimada, conforme ilustra a Figura 8.

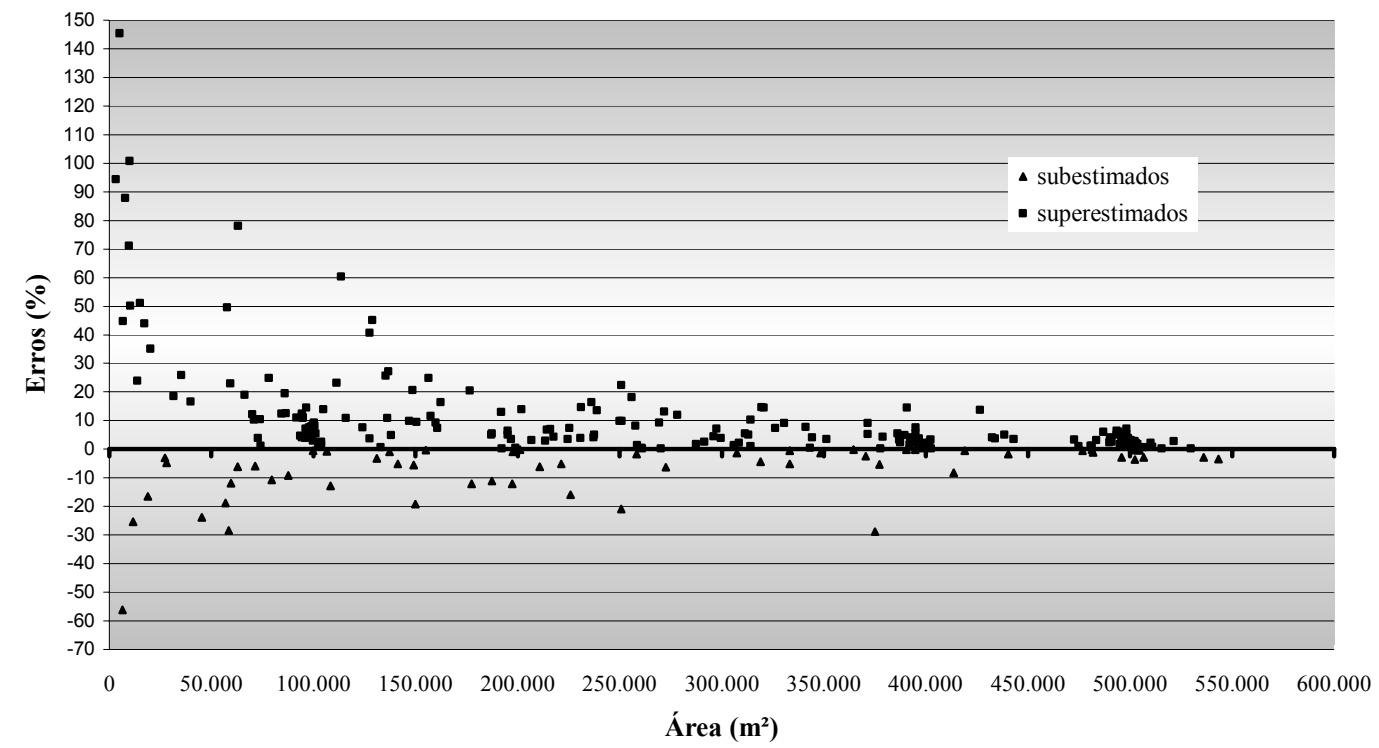

Figura 6. Diagrama de dispersão dos erros cometidos na digitalização em tela da imagem Landsat, considerando todos os 254 talhões.

Figure 6. Scattering diagram of digitizing errors for all 254 stands using Landsat imagery and screen digitizing. 


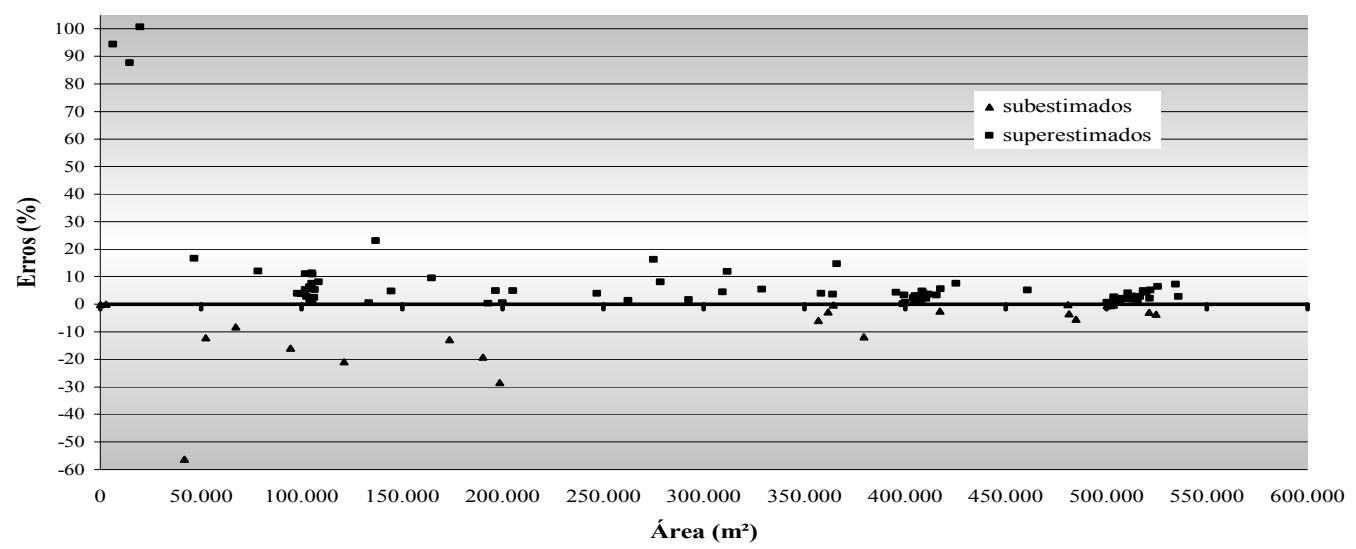

Figura 7. Diagrama de dispersão dos erros cometidos na digitalização em tela da imagem Landsat, considerando os talhões internos.

Figure 7. Scattering diagram of digitizing errors for all internal stands using Landsat imagery and screen digitizing.

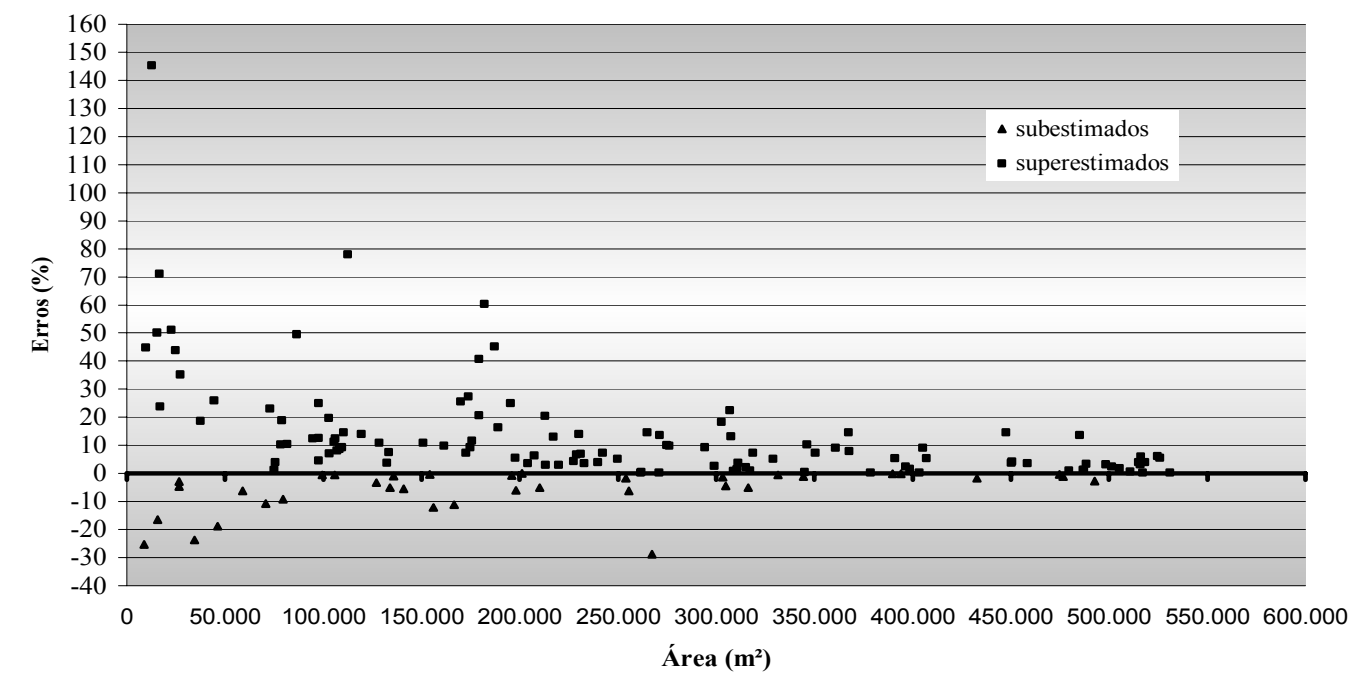

Figura 8. Diagrama de dispersão dos erros cometidos na digitalização em tela da imagem Landsat, considerando os talhões de borda.

Figure 8. Scattering diagram of digitizing errors for all bordering stands using Landsat imagery and screen digitizing.

Manteve-se também uma concentração de freqüências no intervalo de $-10 \%$ a $20 \%$, tanto para os talhões como um todo ou subdivididos nas classes 1 e 2, conforme indica a Tabela 4, sintetizado na Tabela 5, onde os valores foram tomados em módulo. Análise da Tabela 5 mostra que cerca de 48,43\% dos talhões (123) estão na faixa de $0 \%$ a $5 \%$ de erros e $70,48 \%$ dos talhões (179) estão na faixa de 0 a $10 \%$ de erros. Analisando por classe de talhão, os internos apresentam uma freqüência relativa de $61,17 \%$ (63) para o intervalo de $0 \%$ a $5 \%$ de erros e $80,59 \%$ (83) para o intervalo de $0 \%$ a $10 \%$. Já para os talhões de borda, as freqüências relativas seriam de 39,74\% (60) e 63,58\% (96), respectivamente. Comparando-se 
a freqüência relativa desta tabela com a Tabela 3, resultante da digitalização em mesa, observa-se uma certa proximidade dos valores, com os talhões internos (tipo 1), apresentando uma discrepância maior.

Embora a mesa digitalizadora tenha uma precisão superior ao conjunto monitor-mouse, este último apresenta recursos não disponíveis no primeiro, como: possibilidade de variação da escala de trabalho; uso de várias combinações de bandas espectrais; uso de diversos tipos de realce de contraste; uso do alinhamento das estradas em locais com baixo contraste. Outro aspecto é que mesmo sendo de boa qualidade (papel fotográfico Kodak) o papel no qual foi feita a impressão, podem ocorrer distorções na impressão e ele pode também sofrer deformações devido ao manuseio e a condições ambientais. Estes fatores podem justificar os resultados muito similares encontrados em ambos os métodos.

Tabela 4. Distribuição de freqüência dos erros em classes com amplitude de 5\%.

Table 4. Frequency distribution of errors in range class of 5\%.

\begin{tabular}{|c|c|c|c|c|c|c|c|c|}
\hline \multirow{2}{*}{\multicolumn{3}{|c|}{$\begin{array}{c}\text { Classes de Erro } \\
(\%)\end{array}$}} & \multicolumn{3}{|c|}{ Freqüência Absoluta } & \multicolumn{3}{|c|}{ Freqüência relativa (\%) } \\
\hline & & & \multirow{2}{*}{$\frac{\mathrm{TI}}{1}$} & \multirow{2}{*}{$\frac{\mathrm{TB}}{0}$} & \multirow{2}{*}{$\frac{\text { TOTAL }}{1}$} & \multirow{2}{*}{$\frac{\text { TI }}{0,97}$} & \multirow{2}{*}{$\frac{\mathrm{TB}}{0,00}$} & \multirow{2}{*}{$\frac{\text { TOTAL }}{0,39}$} \\
\hline-60 & a & -55 & & & & & & \\
\hline-55 & a & -50 & 0 & 0 & 0 & 0,00 & 0,00 & 0,00 \\
\hline-50 & $\mathrm{a}$ & -45 & 0 & 0 & 0 & 0,00 & 0,00 & 0,00 \\
\hline-45 & a & -40 & 0 & 0 & 0 & 0,00 & 0,00 & 0,00 \\
\hline-40 & $\mathrm{a}$ & -35 & 0 & 0 & 0 & 0,00 & 0,00 & 0,00 \\
\hline-35 & $\mathrm{a}$ & -30 & 0 & 0 & 0 & 0,00 & 0,00 & 0,00 \\
\hline-30 & $\mathrm{a}$ & -25 & 1 & 2 & 3 & 0,97 & 1,32 & 1,18 \\
\hline-25 & $\mathrm{a}$ & -20 & 1 & 1 & 2 & 0,97 & 0,66 & 0,79 \\
\hline-20 & $\mathrm{a}$ & -15 & 2 & 2 & 4 & 1,94 & 1,32 & 1,57 \\
\hline-15 & $\mathrm{a}$ & -10 & 3 & 3 & 6 & 2,91 & 1,99 & 2,36 \\
\hline-10 & $\mathrm{a}$ & -5 & 3 & 8 & 11 & 2,91 & 5,30 & 4,33 \\
\hline-5 & a & 0 & 11 & 20 & 31 & 10,68 & 13,25 & 12,20 \\
\hline 0 & $\mathrm{a}$ & 5 & 52 & 40 & 92 & 50,49 & 26,49 & 36,22 \\
\hline 5 & $\mathrm{a}$ & 10 & 17 & 28 & 45 & 16,50 & 18,54 & 17,72 \\
\hline 10 & $\mathrm{a}$ & 15 & 6 & 20 & 26 & 5,83 & 13,25 & 10,24 \\
\hline 15 & $\mathrm{a}$ & 20 & 2 & 5 & 7 & 1,94 & 3,31 & 2,76 \\
\hline 20 & $\mathrm{a}$ & 25 & 1 & 7 & 8 & 0,97 & 4,64 & 3,15 \\
\hline 25 & $\mathrm{a}$ & 30 & 0 & 3 & 3 & 0,00 & 1,99 & 1,18 \\
\hline 30 & $\mathrm{a}$ & 35 & 0 & 0 & 0 & 0,00 & 0,00 & 0,00 \\
\hline 35 & $\mathrm{a}$ & 40 & 0 & 1 & 1 & 0,00 & 0,66 & 0,39 \\
\hline 40 & $\mathrm{a}$ & 45 & 0 & 3 & 3 & 0,00 & 1,99 & 1,18 \\
\hline 45 & $\mathrm{a}$ & 50 & 0 & 2 & 2 & 0,00 & 1,32 & 0,79 \\
\hline 50 & $\mathrm{a}$ & 55 & 0 & 2 & 2 & 0,00 & 1,32 & 0,79 \\
\hline 55 & $\mathrm{a}$ & 60 & 0 & 0 & 0 & 0,00 & 0,00 & 0,00 \\
\hline 60 & $\mathrm{a}$ & 65 & 0 & 1 & 1 & 0,00 & 0,66 & 0,39 \\
\hline 65 & $\mathrm{a}$ & 70 & 0 & 0 & 0 & 0,00 & 0,00 & 0,00 \\
\hline 70 & $\mathrm{a}$ & 75 & 0 & 1 & 1 & 0,00 & 0,66 & 0,39 \\
\hline 75 & $\mathrm{a}$ & 80 & 0 & 1 & 1 & 0,00 & 0,66 & 0,39 \\
\hline 80 & $\mathrm{a}$ & 85 & 0 & 0 & 0 & 0,00 & 0,00 & 0,00 \\
\hline 85 & $\mathrm{a}$ & 90 & 1 & 0 & 1 & 0,97 & 0,00 & 0,39 \\
\hline 90 & $\mathrm{a}$ & 95 & 1 & 0 & 1 & 0,97 & 0,00 & 0,39 \\
\hline 95 & $\mathrm{a}$ & 100 & 0 & 0 & 0 & 0,00 & 0,00 & 0,00 \\
\hline 100 & $\mathrm{a}$ & 105 & 1 & 0 & 1 & 0,97 & 0,00 & 0,39 \\
\hline 105 & $\mathrm{a}$ & 110 & 0 & 0 & 0 & 0,00 & 0,00 & 0,00 \\
\hline 110 & $\mathrm{a}$ & 115 & 0 & 0 & 0 & 0,00 & 0,00 & 0,00 \\
\hline \multicolumn{3}{|l|}{$>115$} & 0 & 1 & 1 & 0,00 & 0,66 & 0,39 \\
\hline & & To & 103 & 151 & 254 & 100,00 & 100,00 & 100,00 \\
\hline
\end{tabular}

TI = Talhão Interno

$\mathrm{TB}=$ Talhão de Borda 


\section{Avaliação conjunta dos métodos}

A Tabela 6 traz a soma das áreas dos 254 talhões por classe de talhão para o mapeamento tomado como referência (GPS) e as correspondentes obtidas via digitalização da imagem Landsat (em mesa e em tela), bem como a diferença percentual entre elas. Quando se compara os resultados provenientes da digitalização em mesa (coluna 3) com aqueles tomados como referência (coluna 2), verifica-se uma superestimação. Analisando por classe de talhão, através da diferença percentual (coluna 4), observa-se que os talhões classificados como internos apresentaram um erro menor, o que era esperado. Esta maior discrepância para os talhões de borda pode ser justificada lembrando-se que eles apresentaram um maior grau de dificuldade na identificação de seus limites no processo de digitalização. No caso dos resultados oriundos da digitalização em tela este comportamento se mantém. Quando se compara as duas colunas referentes à diferença percentual (4 e 6), verifica-se que a maior discrepância entre as digitalizações em mesa e tela ocorre para a classe dos talhões de borda. Vale ressaltar que a digitalização em tela permite trabalhar com variações de zoom (diferentes escalas) e diferentes tipos de realce de contraste, facilitando a identificação dos limites dos talhões, principalmente em regiões de menor contraste, gerando assim menores erros para os talhões de borda $(4,7 \%)$ quando comparado à digitalização em mesa $(6,0 \%)$.

Tabela 5. Síntese da Tabela 4, com os valores dos erros tomados em módulo.

Table 5. Synthesis of Table 4, with error values in module.

\begin{tabular}{|c|c|c|c|c|c|c|c|c|}
\hline \multirow{2}{*}{\multicolumn{3}{|c|}{$\begin{array}{c}\text { Classes de erro } \\
(\%)\end{array}$}} & \multicolumn{3}{|c|}{ Freqüência Absoluta } & \multicolumn{3}{|c|}{ Freqüência relativa $(\%)$} \\
\hline & & & \multirow{2}{*}{$\frac{T I}{63}$} & \multirow{2}{*}{$\begin{array}{c}\text { TB } \\
60\end{array}$} & \multirow{2}{*}{$\frac{\text { TOTAL }}{123}$} & \multirow{2}{*}{$\frac{\text { TI }}{61,17}$} & \multirow{2}{*}{$\frac{\text { TB }}{39,74}$} & \multirow{2}{*}{$\frac{\text { TOTAL }}{48,43}$} \\
\hline 0 & $\mathrm{a}$ & 5 & & & & & & \\
\hline 5 & $\mathrm{a}$ & 10 & 20 & 36 & 56 & 19,42 & 23,84 & 22,05 \\
\hline 10 & $\mathrm{a}$ & 15 & 9 & 23 & 32 & 8,74 & 15,23 & 12,60 \\
\hline \multirow[t]{2}{*}{15} & $\mathrm{a}$ & 20 & 4 & 7 & 11 & 3,88 & 4,64 & 4,33 \\
\hline & & Total & 96 & 126 & 222 & 93,20 & 83,44 & 87,40 \\
\hline
\end{tabular}

$$
\mathrm{TB}=\text { talhão de Borda }
$$

Tabela 6. Comparação entre as áreas obtidas via digitalização (em mesa e em tela) com a referência, considerando-se os 254 talhões digitalizados.

Table 6. Comparison between areas obtained from tablet and on screen digitizing with the reference, for all 254 digitized stands.

\begin{tabular}{|cccccc|}
\hline Classes & Referência & DM & (Referência - DM) & DT & (Referância - DT) \\
Talhões & Área (ha) & Área (ha) & $(\%)$ & Área (ha) & $(\%)$ \\
\hline Internos & $3.238,6706$ & $3.308,4088$ & 2,2 & $3.305,1600$ & 2,1 \\
De Borda & $3.501,5002$ & $3.711,6328$ & 6,0 & $3.665,7296$ & 4,7 \\
\hline Total & $6.740,1709$ & $7.020,0416$ & 4,2 & $6.970,8896$ & 3,4 \\
\hline
\end{tabular}

$\mathrm{DM}=$ Digitalização em Mesa

DT $=$ Digitalização em Tela

\section{CONCLUSÕES}

$\mathrm{Na}$ digitalização em mesa da imagem Landsat, 43,30\% dos talhões apresentaram erros na faixa de $0 \%$ a $5 \%$ e $64,95 \%$ na faixa de $0 \%$ a $10 \%$ (valores absolutos dos erros).

Na digitalização da imagem Landsat em tela, 48,43\% dos talhões apresentaram erros na faixa de $0 \%$ a $5 \%$ e 70,48\% na faixa de $0 \%$ a $10 \%$ (valores absolutos dos erros).

Para os dois métodos, houve uma forte tendência em superestimar áreas dos talhões.

Não se aconselha o mapeamento de divisas com a metodologia apresentada (digitalização em mesa e em tela), uma vez que elas requerem maior exatidão. 
Levantamentos expeditos ou de reconhecimento podem ser realizados com qualquer uma das metodologias.

Talhões de menores áreas apresentaram erros maiores para os dois métodos utilizados, sendo que os erros seguem uma tendência de queda e estabilização à medida que a área aumenta.

$\mathrm{O}$ erro foi sempre inferior a $6,1 \%$, para qualquer um dos métodos, seja considerando todos os talhões ou suas subdivisões em classes, quando se compararam os totais das áreas obtidas por digitalização com os totais daquelas obtidas por GPS.

\section{REFERÊNCIAS}

GOLFARI, L. Zoneamento Ecológico do Estado de Minas Gerais para Reflorestamento. Belo Horizonte: Centro de Pesquisas do IBDF na Região de Cardoso, 1975. 65 p. (Série Técnica n. 3).

MEDEIROS, A. M. P.; RUDORFF, B. F. T.; SHIMABUKURO, Y. E. Imagens Landsat na estimativa de áreas de cana-de-açúcar, cana e soja. In: SIMPÓSIO BRASILEIRO DE SENSORIAMENTO REMOTO, 8., 1996, Salvador. Anais... Salvador: INPE, 1996. (CD-ROM).

RIBEIRO, C. A. A. S.; VARElla, C. A. A.; SENA Jr., D. G.; SOARES, V. P. Sistemas de Informações Geográficas. Agricultura de precisão. Editado por Aluízio Borém e outros. Viçosa, MG Brasil 2000. 467 p.: il. p-380-407.

SARTORI NETO, A. Subsídios para elaboração do plano de manejo do Parque Nacional Grande Sertão Veredas por meio de um Sistema de Informações Geográficas. Viçosa, 2000. 98 f. . Dissertação (Mestrado em Ciência Florestal) - Universidade Federal de Viçosa,

SKIDMORE, A. K. An expert system classifies eucalipt forest types using thematic mapper data and a digital terrain model. Photogrammetric Engineering and Remote Sensing, vol. 55, n. 10. 1989. p. 14491464.

SOARES, V. P.; HOFFER. R. M. Detecção de mudanças em povoamentos de Eucalyptus spp e outros usos da terra através de imagens TM/LANDSAT-5 na região do vale do Rio Doce-MG. Revista Árvore. Viçosa, MG, v. 20, n. 1. 1996. p.117-127. 